\title{
Application of DSP motion controller in welding robot of oil tank
}

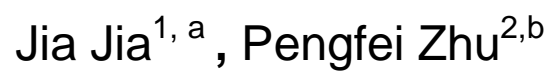 \\ ${ }^{1}$ Kaifeng University, Kaifeng City, Henan Province,China \\ ${ }^{2}$ Kaifeng University, Kaifeng City, Henan Province,China \\ a732387481@qq.com, ${ }^{\mathrm{b}} 544163893 @ q q . c o m$
}

Keywords: Welding robot; Motion controller; Servo control system.

Abstract. In view of design purpose on the welding robot of different tanks, The purpose of this research is to welding robot motion controller is applied to the tank cover, the system adopts solution of "PC + motion controller". The paper analyses the selection of F28335 motion controller, Through the way of multi-axis linkage control, the movement of the manipulator can complete welding of end cover for different type of oil tanks, so as to make full use of features of real-time, high accuracy and good stability on the motion controller.

\section{Introduction}

The demand of crude oil and refined oil in China is growing, the oil tank has not adapted to the development of the times, so the oil tank is developing in the direction of large and diversified. At present, the common oil tank can be divided into cylindrical, square box and spherical, etc. In the welding of the junction between the oil tank and the cover, the difficulty of manual welding and labor intensity is particularly large. The design applies the motion controller to the welding robot in tank cover, it can control the movement of the manipulator by means of multi axis linkage, so as to finish the welding of the end cover for a variety of oil tanks, and realizes the characteristics of real time, high accuracy and good stability of the motion controller.

\section{Overall scheme design of welding robot for shaped oil tank}

Welding robots adopt the master-slave mode, achieved by modules. Speaking from the perspective of control theory, a closed-loop system is composed of the host, motion control system and feedback device. The host (that is personal computer, referred to as PC) sends commands to the motion controller(that is digital signal processing,referred to as DSP), DSP system completes the allocation of instruction, controls the motor of welding torch. In the course of running.Encoder of feedback system collects location information of welding torch, and sents information to the host in order to achieve the control of stepper motor.

The host is a multitasking real-time operating system, it completes the recognition of the current movement of the robot, controls the scheduling of servo motor, processes signal of sensor, manages power system.

Servo control system is the lower computer system, controls the speed, position or torque of motor rotation, processes problem in the process of running. This system completes digital control of the motor process based on DSP (Digital Signal Processing) as the core control device.

\section{Hardware design of welding robot for shaped oil tank}

Welding of oil tank cover is a complicated process. In this design, the welding trajectory is determined according to the shape of the end cover, the tank body is at rest in the meantime.

Three-dimensional model of welding robot is built by UG parametric software, as shown in Fig. 1. The structure consists of 4 axes: 1 axis realizes vertical movement; 2 axis achieves horizontal movement; 3 axis completes the front and rear movement of the work platform, it can confirm and 
correct the position of the working table relative to the oil tank body; 4 axis completes attitude adjustment in motion of the welding torch.

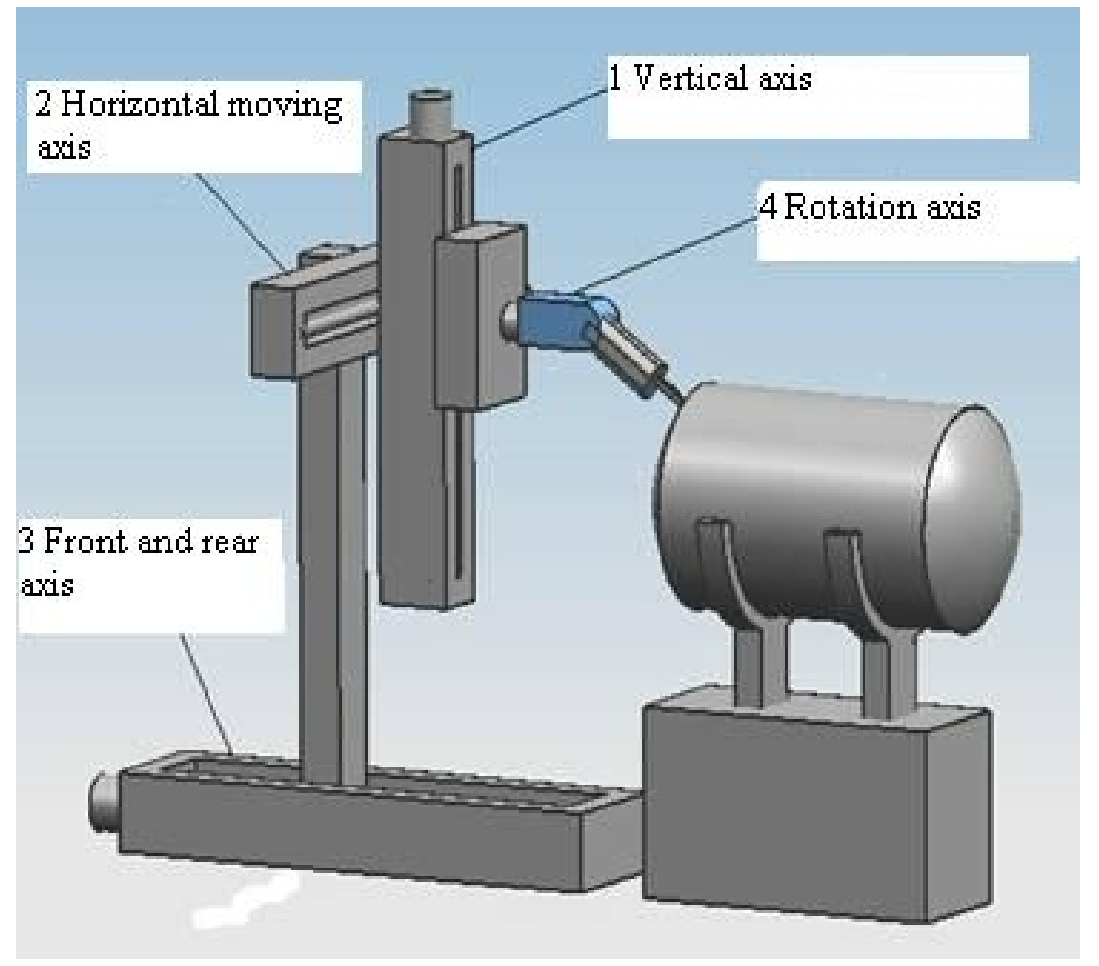

Fig. 1 Three-dimensional model of welding robot

Firstly, the angle of welding torch is determined during the welding process, it can be rotated by adjusting the device. Tilt angle is $15^{\circ} \sim 25^{\circ}$, which is conducive to weld forming. The technological parameter in welding process comprises welding current(420 180 A), welding voltage(24 28V), welding speed(450 700 $\mathrm{mm} / \mathrm{min})$.

\section{Software design of welding robot for shaped oil tank}

As the upper computer, personal computer provides control functions to consumer using a plurality of dynamic link library. These functions are divided into self check, initialization, setting of environmental parameters, selection and setup of motion parameters, motion control command, I/O operation of users, acquisition of status, error handling, etc. Corresponding to these library functions, driver software control motion controller, subroutine section of DSP completes concrete operation.

The software of the lower computer includes the realization of the program of DSP and the control algorithm. This system uses TMS320F28335 control chip as the digital signal processor. The chip maintains the advantages of the original DSP, and it can realize difficult floating point operation, store large amounts of data and programs, calculate exactly.

In the course of operation, the internal power supply voltage of the chip is $1.9 \mathrm{~V}$, the external power supply is $3.3 \mathrm{~V}$. It Not only reduces the power consumption of the control chip, but also increases the use time of the chip. Such as division, trigonometric functions, and FFT are often used in the field of industrial control, the operating speed of floating point device is $2 \sim 3$ times faster than the fixed device.

The functional block diagram of DSP is shown in Fig. 2. 


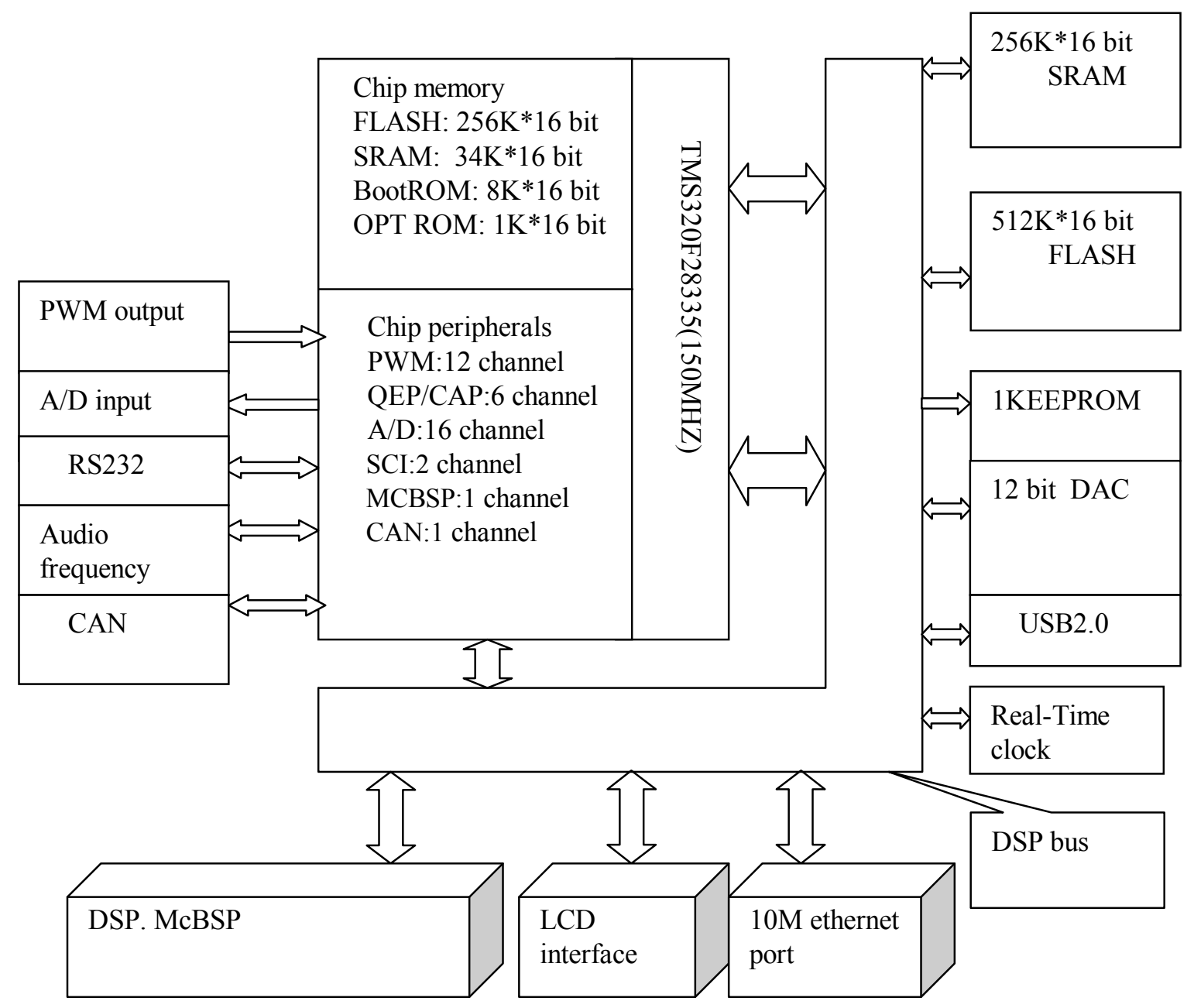

Fig. 2. Functional block diagram of DSP

TMS320F28335 integrates a lot of peripherals, it does not contain EV module,instead, three new peripheral modules ePWM, eCAP and eQEP. EPWM module is the enhanced PWM peripherals, supports the generation of independent / complementary PWM. In order to achieve more accurate control effect, the hardware expansion module is added, that is high precision pulse width modulator (HRPWM). eCAP module provides complete capture channel, completes multiple time capture tasks according to the requirements of target task. If the snap snap is not working at the input capture state, a single channel pulse width modulation (PWM) generator can be formed with the eCAP module resource. As the enhanced orthogonal pulse code module, eQEP contains the position detector and the orthogonal edge snap detection unit of the low speed measurement.

In the process of the stepper motor driver, if the pulse frequency is not normal,the motor will stall, and can't drive load. The method of adding and deceleration control to the stepping motor is mainly controlled by the control algorithm, S type curve acceleration and deceleration control, exponential control method. The acceleration and deceleration control of the motor is very different from the actual acceleration and deceleration process of the motor. When the motor is set at the speed of the change, there is noise and poor stability. The algorithm of S type curve acceleration and deceleration control is complex, it is hard to realize in application;Exponential control method accords with change law of speed in the operation of stepper motor largely.

There are three stages in the operation of the stepping motor: acceleration, uniform speed and deceleration movement. The timer is configured as a continuous incremental count mode during acceleration, each velocity value is converted to the corresponding pulse frequency, so as to get value of the periodic register in the control chip F28335 timer. Stepper motor runs smoothly after the acceleration phase, this point is the uniform state, the value of the corresponding periodic registers in the control chip is also increased, the chip also stores information of number for each operation step. When the interrupt subroutine runs, step number of stepper motor began to decrease, the timer is 
configured to continuous decline model. After the completion of each operation, the system will enter the next paragraph of information processing while loading the value of the timer in the periodic register.

It is not dependent on the absolute value of the amount of control, but on the added value of the amount of control in the stepping motor control. It is necessary to use PID incremental algorithm, avoiding accumulation of past data in the process of position operation. As a result of the improvement of control algorithm, it reduces the interference to minimum because of wrong operation, and more simple and feasible than general PID control algorithm.

The development environment of this system is CCS3.3, as shown in Fig. 3. It is developed by Texas Instruments company for the characteristics of DSP motion controller. Writting and compiling, debugging, link and download of program can be completed in the same window, it is easy and intuitive to use, shortens the development time of the program to a great extent .

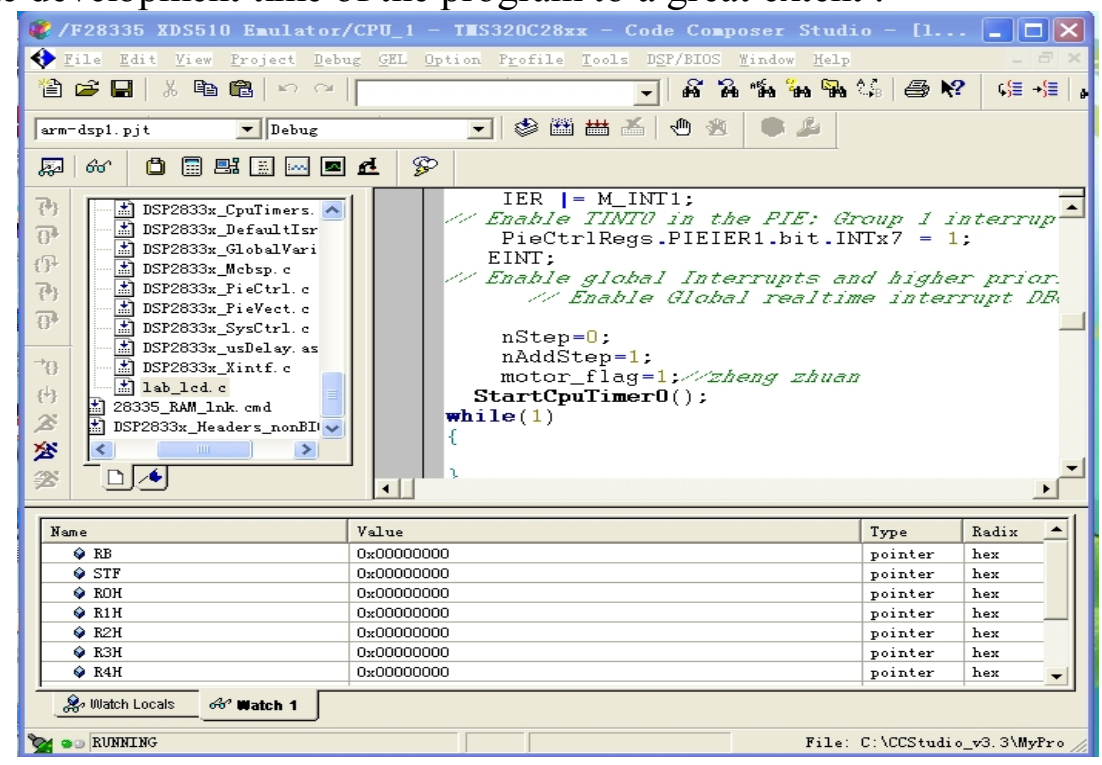

Fig. 3 CCS3.3 development environment

\section{Conclusions}

This design uses the idea of "PC+DSP motion controller". The real-time control of the motion controller is combined with the welding robot of the shaped oil tank, which ensures the real-time requirements of servo control system. For the different shapes of oil tanker in production, this system realizes the opening of welding for different oil tanks in the process of manufacturing. It reduces the interference to minimum because of wrong operation, and is more convenient than the previous control.

\section{References}

[1]Anfu Li, Qingfei Wang, Xiaohong Li. Design of digital controller based on TMS320C+32[J].Electric Power Automation Equipment, 2011,26(1):55-57.

[2]Wenqiang Wang, Ping He, Lei Cui, Zhicheng Wang, Sijie Bi. Design and implementation of 32 bit servo controller for numerical control[J].Chinese Computer Systems,2010,31(7):1413-1415.

[3]Shihe Yang, Jinglin Liu, Jinping Zhang, Shuaifu Wang. Design of multi motor cooperative control system based on DSP[J]. Measurement \& Control Technology,2010,29(6):45-47.

[4]Mancun Gui. Research and development of motion control card based on DSP[D].Shanghai:Shanghai Jiao Tong University,2010. 